ESAIM: PROCEEDINGS AND SURVEYS, December 2017, Vol. 58, p. 98-117

Stéphane DELLACHERIE, Gloria FACCANONI, Bérénice GREC, Frédéric LAGOUTIERE, Yohan PENEL

\title{
HIGH TEMPERATURE THERMAL HYDRAULICS MODELING OF A MOLTEN SALT: APPLICATION TO A MOLTEN SALT FAST REACTOR (MSFR)*,**
}

\author{
Pablo R. Rubiolo ${ }^{1,2}$, Mauricio Tano Retamales ${ }^{1}$, Véronique Ghetta ${ }^{1}$ and \\ JuLien GiRAud ${ }^{1}$
}

\begin{abstract}
An overview of the ongoing efforts in the area of the thermal hydraulics modeling of a Molten Salt Fast Reactor (MSFR) is presented. The MSFR employs a flowing liquid fuel based on a high temperature lithium fluoride salt. A molten salt flow can be considered in many situations as an incompressible flow. However, several phenomena intrinsic to a molten salt flow pose unique challenges (radiative heat transfer, volumetric heat source, strong neutronics feedbacks, etc.). To study some of these phenomena and to improve current Computational Fluid Dynamics (CFD) models an experimental facility called SWATH (Salt at WAll: Thermal ExcHanges) will be built as part of the European project SAMOFAR (2015-2019).
\end{abstract}

Résumé. Une description des efforts en cours dans le domaine de la modélisation thermo hydraulique du réacteur à sel fondu rapide (Molten Salt Fast Reactor, MSFR) est présentée. Le MSFR utilise un combustible nucléaire liquide basé sur un sel fondu de type Lithium Fluorure (LiF). Dans la plupart des conditions d'opération normale ou accidentelle un sel fondu peut être considéré comme étant un fluide incompressible. Néanmoins la modélisation précise de plusieurs phénomènes intrinsèques au sel fondu pose un important défi (transfert radiatif, source volumétrique de puissance, contreréactions neutroniques, etc.). Dans le cadre du projet Européen SAMOFAR (2015-2019), la plateforme expérimentale SWATH (Salt at WAll : Thermal ExcHanges) est actuellement en construction. Cette plateforme permettra d'étudier certains de ces phénomènes et ainsi d'améliorer la précision des modèles de type Computational Fluid Dynamics (CFD) utilisés pour prédire les écoulements du sel combustible.

\section{INTRODUCTION}

Molten Salt Reactors have attracted increased attention in recent years because of the design and safety possibilities offered by the use of a liquid fuel. The European project SAMOFAR (2015-2019) is currently coordinating the research efforts on the Molten Fast Salt Reactor (MSFR) concept in Europe. The MSFR [19] is a fast-spectrum breeder reactor with a large negative power coefficient ${ }^{1}$ that can be operated in a Thorium fuel

\footnotetext{
* This project has received funding from the Euratom research and training program 2014-2018 under grant agreement No 661891.

** The content of this article does not reflect the official opinion of the European Union. Responsibility for the information and/or views expressed in the article lies entirely with the authors.

1 LPSC, University of Grenoble-Alpes, CNRS/IN2P3, 53 rue des Martyrs, 38026 Grenoble, France.

2 e-mail: pablo.rubiolo@lpsc.in2p3.fr

${ }^{1} \mathrm{~A}$ negative power coefficient contributes to the reactor stability since it will oppose any reactor power change.
}

(c) EDP Sciences, SMAI 2017 


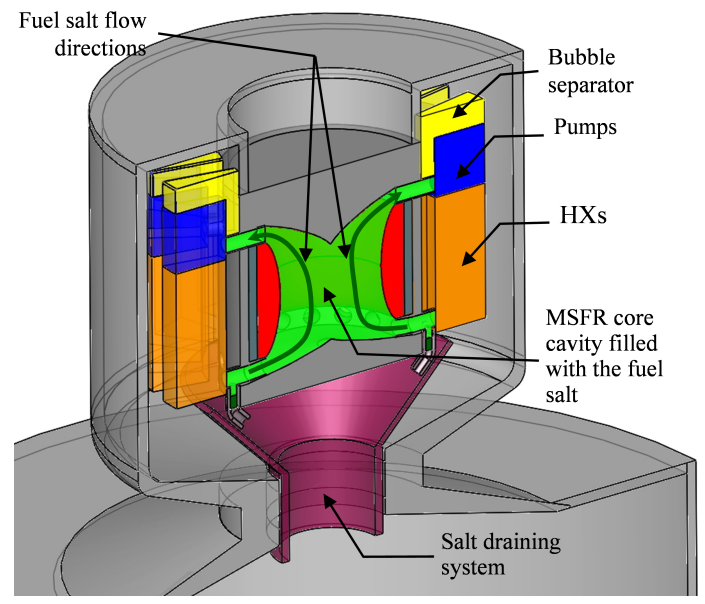

FIgURE 1. Approximated layout of the Molten Salt Fast Reactor (MSFR) fuel circuit.

cycle. The fast ${ }^{2}$ spectrum allows the reduction of the reprocessing requirements and a better reactor breeding ratio $^{3}$. Some of the advantages [23] of the MSFR design are the possibility for actinide burning and extending fuel resources, on-line fuel loading and reprocessing and the use of novel passive safety systems such as the fuel salt draining system.

The reference MSFR concept is a $3000 \mathrm{MW}(\mathrm{th})$ reactor with three different circuits: the fuel circuit, the intermediate circuit and the power conversion system. Figure 1 presents the schematic layout of the Molten Salt Fast Reactor (MSFR). The main components of the fuel circuit are the fuel salt which serves as fuel and coolant, the core cavity, the inlet and outlet pipes, the gas injection system (not shown in the figure), the salt-bubble separators, the fuel Heat Exchangers (HXs) and the pumps. A lithium fluoride (LiF) salt has been selected as the fuel matrix. The initial composition (non-irradiated) of the MSFR fuel salt is a mixture of a lithium fluoride, thorium fluoride and actinides fluoride salts $\left(\mathrm{LiF}_{-} \mathrm{ThF}_{4^{-}}{ }^{233} \mathrm{UF}_{4}\right.$ or $\left.\mathrm{LiF}_{-} \mathrm{ThF}_{4^{-}}{ }^{e n r} \mathrm{UF}_{4}-(\mathrm{Pu}-\mathrm{MA}) \mathrm{F}_{3}\right)$, with the proportion of $\mathrm{LiF}$ fixed at about $77.5 \%$. The total fuel salt volume in the fuel loop is about $18 \mathrm{~m}^{3}$ and the mean core salt temperature is approximately $725^{\circ} \mathrm{C}$. As shown in Figure 1, the fuel salt flows from the bottom to the top of the core cavity. After exiting the core, the fuel salt is fed into 16 groups of pumps and Heat Exchangers (HXs) located around the core. The fuel salt circulates in the fuel circuit in around 3-4 seconds. The fuel circuit includes a salt draining system which can be used for a planned reactor shut down or in case of incidents/accidents leading to an excessive increase of the temperature. This is an unique characteristic of a liquid fuel which allows for draining the fuel salt using gravity forces (passive system) into dedicated salt draining tanks where the fuel can be cooled without returning to neutron criticality thanks to the draining tanks volume and geometry.

Three other components of the MSFR core are: the upper and lower axial neutron reflectors and the radial fertile ${ }^{4}$ blankets (shown in red in Figure 1). The neutron reflectors allow reducing the neutron leaks from the core and also provide neutron shielding for the structures outside the core. The radial fertile blankets are part of the radial reflector and their purpose is to increase the MSFR breeding ratio (i.e. the production of new fissile material that can be used as fuel for the reactor). The reactor blanket is filled with the fertile

\footnotetext{
${ }^{2}$ In a fast reactor neutrons are not slowed down by a moderator and the chain reaction is sustained by high kinetic energy neutrons.

3 The breeding ratio is defined as the number of fissile atoms produce per fission. The MSFR has a breeding ratio slightly greater than one meaning that the reactor produces more fuel than what it is consumed

${ }^{4}$ Fertile nuclei (examples are ${ }^{232} \mathrm{Th},{ }^{238} \mathrm{U}$ and ${ }^{240} \mathrm{Pu}$ ) are not fissionable by thermal neutrons but can be converted into a fissile nuclei after a neutron capture. On the contrary, fissile nuclei are fissionable by thermal neutrons (examples are ${ }^{233} \mathrm{U},{ }^{235} \mathrm{U},{ }^{239} \mathrm{Pu}$ and $\left.{ }^{241} \mathrm{Pu}\right)$.
} 
salt $\mathrm{LiF}_{-}{ }^{232} \mathrm{ThF}_{4}$ with an initial composition of $22.5 \%$ mole ${ }^{232} \mathrm{ThF}_{4}$. Two important aspects of the MSFR are the low core reactivity inventory and the absence of control rods or neutron poisons. The first one is achieved thanks to the online fueling and reprocessing. The second one is possible due to the large negative reactivity feedback coefficients (Doppler and fuel density effects) which allow to control the core reactivity only based on the natural reactor response due to the balance between the power generated in the core and the power extracted in the HXs. For instance during accidents leading to an insufficient heat removal in the HXs or a sudden large reactivity insertion, the Doppler and fuel salt density feedback effects bring the reactor to a subcritical state nearly instantaneously (thus more efficiently than a mechanical neutron absorbent rods system) and with a limited increase of the fuel temperature [15]. The principal challenge in these situations is the nuclear decay heat released in the fuel salt that requires maintaining adequate cooling of the fuel salt in the core cavity or to drain the fuel salt from the core cavity to salt storage tanks in a few minutes to avoid an excessive temperature rise. In the MSFR absorbent rods are not either needed for power shape control since the fuel salt circulation ensures a homogenous fuel irradiation and there are not design or safety criteria imposing limits on the power shape like in nuclear reactors using solid fuel such as Pressurized Water Reactors (PWRs), Boiling Water Reactors (BWRs) and Sodium Fast Reactor (SFRs). The absence of absorbent rods simplifies the reactor operation and eliminates some possible accident initiators (e.g. a neutron absorbent rod ejection).

Lastly, another key reactor component is the fuel reprocessing unit ${ }^{5}$ (not shown in the figure) [6]. Thanks to the MSFR fast spectrum, this unit only extracts a small amount of the fuel salt (a few liters per day) for fission products removal and then returns the cleaned fuel salt to the reactor. This is an improvement over thermal spectrum Molten Salt Reactors (MSRs) which usually require large reprocessing salt volumes making more challenging the reactor operation.

As discussed in the previous paragraphs, the MSFR design has some potential advantages and also poses new challenges in terms of reactor design and safety that need to be evaluated. The existence of several phenomena inherent to molten salts that do not exist in other reactors types requires the development of better adapted numerical models and also of a new methodology to perform the safety evaluation. Development of accurate numerical models for the MSFR can only be accomplished by the use of a multiphysics approach which allows taking into account the strong coupling existing between neutronics, thermal hydraulics and thermomechanics phenomena in the reactor. As it is discussed in the next sections, such numerical reactor model requires further progress on the knowledge of molten salt thermal hydraulics and on the development of fuel salt Computational Fluid Dynamics (CFD) models. The need of experimental data to evaluate these more adapted CFD models is one of the motivations for the SWATH (Salt at WAll: Thermal ExcHanges) experiment which is described later in this paper. Before discussing current efforts on molten salt thermal hydraulics, a description of the multiphysics approach adopted for this reactor is provided in Section 1. Section 2 provides an overview of some of the challenges related to (a) the understanding and the numerical modeling of the thermal hydraulics phenomena and (b) the experimental techniques required to study them. Finally, the ongoing CNRS thermal hydraulics experimental activities are presented in Section 3.

\section{Towards a Multiphysics Model of the MSFR}

As other fission nuclear reactors, energy is produced in the MSFR fuel through the nuclear fission process which is schematized in the Figure 2. As shown in this figure, a fission reaction produces approximately $200 \mathrm{MeV}$ of energy and between one to three neutrons. Neutrons in the reactor disappear by three main mechanisms:

- Radiative capture (for example a neutron capture in a fertile material such as thorium),

- Leaks from the reactor core,

- New fission after a neutron absorption in a fissile material ( $\operatorname{such}$ as ${ }^{233} U$ ).

In order to maintain the reactor neutron population stable over time, exactly "one" neutron per fission has to be kept in average to induce a new fission. Given the extremely short value of the neutron mean lifetime in a reactor (of the orders of a few microseconds) any small deviation from the objective of one neutron per fission

\footnotetext{
${ }^{5}$ The reprocessing unit allows for recovering fissile material and actinides from the spent fuel salt.
} 


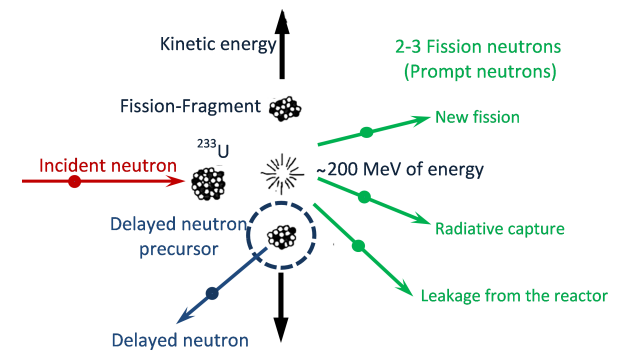

(a) Fission process

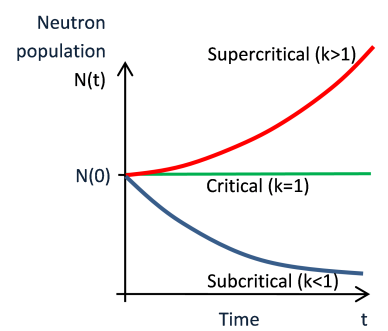

(b) Reactor response

Figure 2. The nuclear fission process. Evolution of the total neutron population in function of the reactor multiplication factor $\mathrm{k}$.

causes a very rapidly increase or decrease of the neutron population. The dynamic state of a nuclear reactor is usually described by the Reactor Multiplication Factor $k$ which can be defined as follows:

$$
k=\frac{\text { Number of Neutrons at the Generation } i+1}{\text { Number of Neutrons at the Generation } i}
$$

As can be seen on the right side of Figure 2 when the multiplication factor $k$ is equal to one, the neutron population remains stable and the reactor power does not change. If $k$ is greater than one, neutron population increases at each generation. In this case, the reactor population diverges exponentially and the reactor is in supercritical state. On the contrary, if $k$ is less than one, the neutron population decreases exponentially to zero and the reactor is in subcritical state. Equation 1 provides a very intuitive definition of the reactor multiplication factor. Nevertheless it is rather difficult to calculate $k$ from it. The multiplication factor $k$ can be alternatively defined as follows:

$$
k=\frac{\text { Neutrons Production Rate }}{\text { Neutron Absorption Rate }+ \text { Neutrons Leak Rate }}
$$

On the contrary to the first definition of the reactor multiplication factor, Equation 2 provides a more practical way for the evaluation of $k$ since the neutrons production, absorption and leaks rates can be calculated from the nuclear reaction cross sections of the reactor materials and the neutron flux in the reactor.

As shown in Figure 2, two different types of neutrons result from the nuclear fission process: prompt and delayed neutrons. Prompt neutrons are emitted by the primary fission fragments nearly immediately (within $10^{-14} \mathrm{sec}$ ) after the fission event. On the contrary, delayed neutrons are emitted by a small fraction of fission fragments called the effective delayed neutron precursors which have already undergone a beta decay chain. The emission of the delayed neutrons is therefore delayed by the time constant associated to the beta decays which can range from a few milliseconds to several seconds. The delayed neutrons are then emitted orders of magnitude later than the prompt neutrons. The delayed neutron fraction ${ }^{6}$ strongly depends on the fissile material and it is very small (less than 1 percent for nuclei such as ${ }^{233} U,{ }^{235} U$ and ${ }^{239} P u$ ). Nevertheless, delayed neutrons are very important for reactor control since they allow to increase the effective neutron mean lifetime in the reactor from tens of microseconds to tens of milliseconds. For transients with relatively moderate unbalance between the neutron production and destruction terms, the reactor time response is therefore sufficiently slowed down by this effect to allow for the use of mechanical control systems.

At this point it is important to notice that the overall effect of delayed neutrons in a nuclear reactor using a liquid fuel such as the MSFR is different from the one that exists in a solid fueled reactor (PWR, BWR, SFR,

\footnotetext{
${ }^{6}$ The delayed neutron fraction $\beta[7]$ is defined as the ratio of the average number of delayed neutrons $\nu_{d}$ produced per fission to the average total number of neutrons produced per fission (i.e. the sum of the prompt neutrons number $\nu_{p}$ plus the delayed neutrons number $\left.\nu_{d}\right): \beta=\frac{\nu_{d}}{\nu_{p}+\nu_{d}}$.
} 


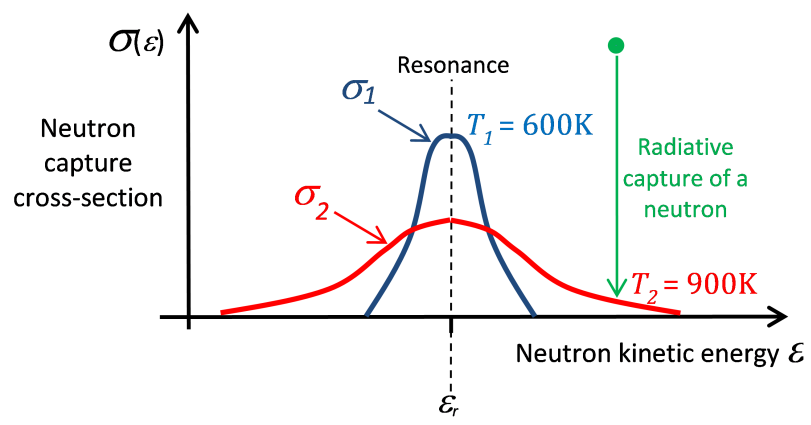

Figure 3. Thermal Doppler broadening of a resonance for the neutron capture cross sections of a fertile nucleus. When the fuel temperature increases from $T_{1}$ to $T_{2}$, the resonance becomes wider and a neutron that, at $T_{1}$ was outside the resonance, at $T_{2}$ falls within the resonance and can be captured.

etc.) because in the MSFR the delayed neutron precursors flow with the molten fuel salt. Therefore, on the contrary to solid fueled reactors, delayed neutrons are not emitted at the place where the fission occurred but at a distance that depends on the fuel salt velocity flow field. Moreover since the influence of delayed neutrons on the reactor transient response depends on the place where they are emitted ${ }^{7}$ then the impact of the delayed neutrons will depend on the fuel salt flow structure and will vary with a flow change. For example, a sudden increment of the fuel salt flow will cause a higher fraction of the delayed neutron precursors to decay outside the core cavity. This will slightly decrease the neutron production rate (i.e. the reactor multiplication factor) and the mean neutron lifetime (i.e. shortening the reactor response time). Accurate prediction of the reactor transient behavior requires thus calculation of the delayed neutron precursors concentration based on the fuel salt flow field and the fission rate.

Beside the effect of delayed neutrons, another important phenomena in reactor physics are the reactivity feedback effects[7] which contribute to the stabilization of the reactor power. In the case of the MSFR there are two main reactivity feedback effects: salt density and Doppler effects. By design, both reactivity feedback effects are strongly negative in the MSFR and accordingly they oppose the reactor power variations. Salt density effect is related to the change on the nuclear reactions macroscopic cross-sections due to the changes of the concentrations of the nuclides composing the fuel salt (as density change). The salt density feedback is negative since a reduction of the fuel salt density (for example following a rise of the salt temperature) increases the neutron mean free-path and thus the neutron leaks, causing a decrease of the multiplication factor (see Equation 2). The Doppler effect arises from the dependency of the neutron cross sections with respect to the relative velocity between the neutron and the nucleus and causes a broadening of the neutron absorption resonances as the fuel temperature rises such as it is illustrated in the example of Figure 3 . The broadening of the resonances increases the neutrons absorptions, in particular in the fertile nuclei, and results in a rapid reduction of the reactor multiplication factor. The Doppler effect provides thus a strong and almost instantaneous negative feedback on the neutron population variation rate that will oppose to the temperature (and power) changes. In the same manner as for the delayed neutron precursors, determination of these two reactivity feedback effects (Density and Doppler) requires a good knowledge of the temperature field in the fuel and thus an accurate thermal hydraulics modeling of the molten fuel salt.

Due to these reactivity feedback and delayed neutron effects, any accurate model of a liquid fueled nuclear reactor has to take into account the coupling between the thermal hydraulics and neutronics phenomena. As schematized in Figure 4, the thermal hydraulics model determines the fuel salt temperature and velocity fields

\footnotetext{
${ }^{7}$ A delayed neutron emitted near the center of the core has higher chance to induce a new fission than a delayed neutron emitted near the periphery of the core (where the probability of leaking is higher) and therefore will have a larger effect on the reactor dynamic behavior. This phenomenon is called neutron importance.
} 


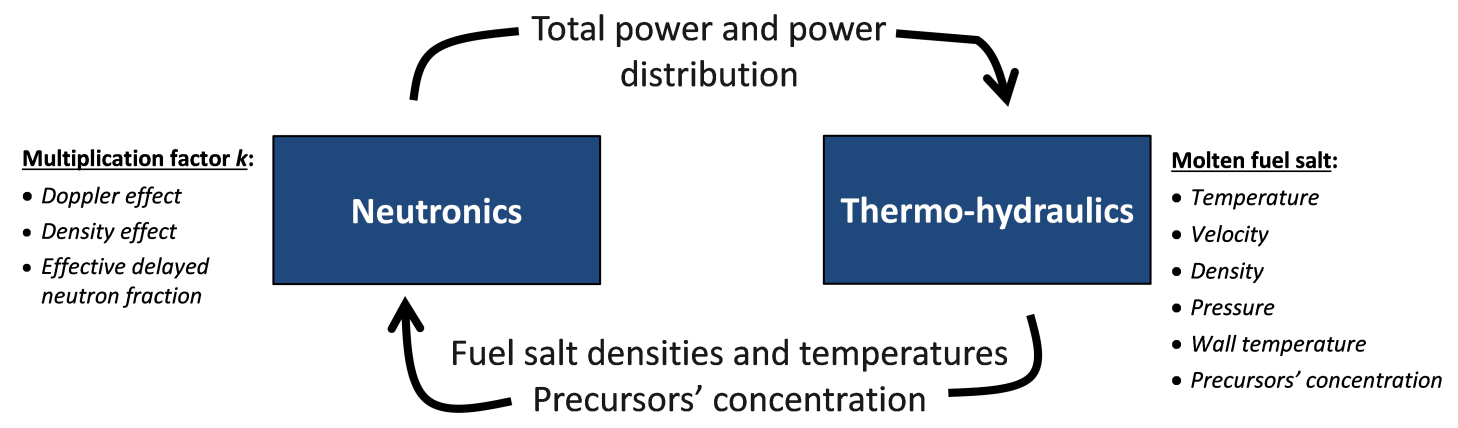

FIgURE 4. Simplified schema of the neutronics and thermal hydraulics phenomena coupling existing in a Molten Salt Reactor.

according to the reactor nuclear power. These two fields are used to calculate the fuel salt densities and the delayed neutron precursors concentrations which are then transferred to the neutronics model. The neutronics model uses them to determine the reactor multiplication factor (via the reactivity feedback effects: density and Doppler effects) and the effective delayed neutron fraction. The neutronics model recalculates then the total reactor power and the power distribution based on the neutronics flux. The numbers of data exchanges between the neutronics and thermal hydraulics models depends on the type of calculation that is being performed but the information exchanged is similar for steady state or transient analyses.

Attention is now turn to the thermal hydraulics modeling. As can be seen in Figure 1, the MSFR core cavity is a rather complicate geometry with curved walls and sixteen inlet and outlet channels. This shape has been chosen to improve the flow mixing inside the cavity and to decrease the salt hot spots. Due to the complexity of fuel salt flow inside the cavity, relatively simple hydraulics models such as subchannels or porous medium models commonly used for Pressurized Water Reactors (PWRs) fuel assemblies are not accurate enough. Only the more precise numeric modeling provided by a Computational Fluid Dynamic (CFD) code is suitable to study the flow phenomena inherent to this reactor:

- 3D flow effects in the core cavity,

- Strong turbulent flow (for instance Reynolds number is greater than 500,000 in the core cavity),

- Presence of flow recirculations that can have a significant impact on the core wall temperatures,

- Strong coupling between the thermal hydraulics and neutronics phenomena.

For the ongoing MSFR studies, the CFD code OpenFOAM (Open source Field Operation And Manipulation)[10] has been employed to solve the fuel salt thermal hydraulics equations. OpenFOAM[11] is an open source toolbox that allows developing (and provide) numerical solvers, and pre-/post-processing utilities for the solution of Computational Fluid Dynamics (CFD). The geometry domain solved in the CFD simulations usually includes the main fuel circuit components such as the core cavity, the inlet/outlet channels and the Heat Exchangers (HXs). A detailed model of the fuel salt pumps is not necessary in many cases since the reactor pumps can be taken into account as a fixed pressure rise before the entrance of the HXs without introducing a significant error. Moreover for most transient studies, the HXs can be approximated as a porous medium with a fixed porosity and permeability tensor (see discussion in Section 2.1) to reduce computational cost. The MSFR bubbling system used for fission product reprocessing is not modeled either since, under most situations, it has a small impact on the salt flow. If one does not intend to study the effect of a flow unbalance between the MSFR loops or other type of interactions between loops, a one-sixteen core model can be used (with appropriate boundary conditions) in the thermal-hydraulics simulations as can be seen in the CFD mesh presented in Figure 5.

Several possible CFD approaches can be used to solve the fuel salt thermal hydraulic equations. The more common are: 


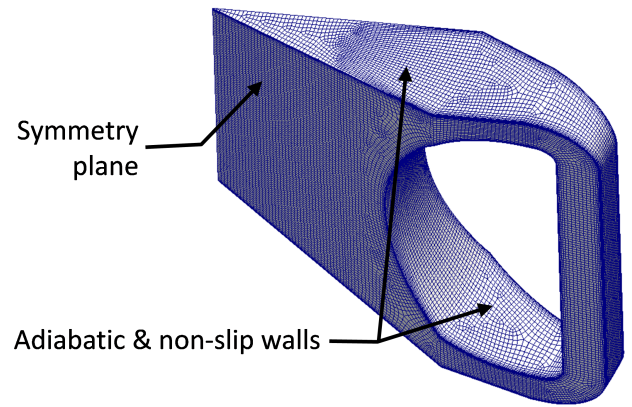

Figure 5. Example of 1/16 MSFR core model for a CFD calculation (the mesh in the figure contains 300,000 cells).

- Reynolds-averaged Navier-Stokes equations (RANS): this approach uses the time-averaged equations of motion for fluid flow (continuity, Navier-Stokes and energy). Time averaging is performed on a large time scale so turbulence is filtered out.

- Direct Numerical Simulation (DNS): computational fluid dynamics simulation in which the Navier-Stokes equations are numerically solved without any turbulence cloture model. Computational mesh should allow to resolve all significant scale of turbulence.

- Large Eddy Simulation (LES): family of methods that make a compromise between RANS and DNS. Large-scale eddies are resolved in the flow equation solution while the effects from the small-scale eddies are determined from dedicated models (low-pass filtering).

- Detached eddy simulation (DES): further compromise between RANS and LES, to capture key physical phenomena in the lowest possible amount of computer time. This approach consists in a modified RANS model which switches to a subgrid scale formulation in regions where a LES calculations is needed.

This list is not exhaustive and other CFD approaches exist. Given that ultimate goal is to develop a multiphysics model of the main reactor components including not only thermal hydraulic phenomena but also neutronics and thermomechanics, from the above approaches, only the RANS or the DES approaches provide at this time a reasonable compromise between accuracy and computational effort. The mesh requirements of DNS and LES approaches preclude their practical implementation in our applications. In the present work a RANS approach has been favored to solve the flow mass, momentum and energy conservation equations [14].

The equations of the RANS model for the fuel salt conservation equations are usually written assuming incompressible flow as follows:

$$
\begin{gathered}
\frac{\partial \overline{u_{j}}}{\partial x_{j}}=0 \\
\frac{\partial \overline{u_{i}}}{\partial t}+\frac{\partial \overline{u_{i}} \overline{u_{j}}}{\partial x_{j}}=-\frac{\partial}{\partial x_{i}}\left(\frac{\bar{p}}{\rho_{0}}+\frac{2}{3} k\right)+\frac{\partial}{\partial x_{j}}\left\{\left(\nu+\nu_{t}\right)\left[\left(\frac{\partial \overline{u_{i}}}{\partial x_{j}}+\frac{\partial \overline{u_{j}}}{\partial x_{i}}\right)-\frac{2}{3} \frac{\partial \overline{u_{k}}}{\partial x_{k}} \delta_{i j}\right]\right\}+g_{i}\left[1-\beta_{\text {salt }}\left(\bar{T}-T_{0}\right)\right] \\
\frac{\partial \bar{T}}{\partial t}+\frac{\partial \bar{T} \overline{u_{j}}}{\partial x_{j}}=K_{\text {eff }} \frac{\partial}{\partial x_{k}} \frac{\partial \bar{T}}{\partial x_{k}}+S
\end{gathered}
$$

where $x_{i}$ is the $\mathrm{i}$-component of the position vector, $\overline{u_{j}}$ the time-averaged $\mathrm{j}$-component of the flow velocity, $\bar{p}$ the time-averaged flow pressure, $\rho$ is the fluid density, $k$ is the turbulent kinetic energy, $\nu$ the fluid kinematic viscosity, $\nu_{t}$ the fluid turbulent kinematic viscosity, $\delta_{i j}$ the delta de Kronecker function, $g_{i}$ the i-component of the gravity acceleration, $\beta_{\text {salt }}$ the fuel salt temperature expansion coefficient, $\bar{T}$ the time-averaged flow temperature, $T_{0}$ a reference temperature, $K_{\text {eff }}$ the flow effective thermal diffusivity including the turbulence effect and $S$ is the heat source inside the flow. 
The molten salt flow equations 3 to 5 are derived using the Boussinesq approximation. This approximation neglects the effects of fluid density variations except in the gravitational force term of the linear momentum conservation equation 4. The Boussinesq approximation also treats the other fluid properties as constant. Accordingly, the molten salt is considered as an incompressible flow with an equivalent buoyant force estimated from the salt thermal expansion coefficient $\beta_{\text {salt }}$ and temperature variation. The use of the Boussinesq approximation is justified during reactor nominal conditions and also in many accidental transients because:

- The fuel salt flow Mach number is very small. Indeed nominal molten fuel salt velocities in the MSFR are comprised between $1-4 \mathrm{~m} / \mathrm{s}$ resulting in a small enough Mach number $(M a=U / c \ll 1$ where $c \mathrm{~s}$ the speed of sound) that allows neglecting the fuel salt density variations caused by pressure changes.

- The presence of pressure waves with large amplitudes is not expected except in some very hypothetical scenarios.

- The vertical scale $L$ of the flow circuit in the reactor is very small (only a few meters) making the density changes due to hydrostatic pressure variations also negligible $\left(L \ll c^{2} / g\right.$ where $g$ is the gravitational acceleration).

- The effects of the fuel salt density changes caused by temperature variations alone can also be neglected in the continuity equation. This is in general the case at reactor nominal conditions and also in most accidental transients. If one assumes a flow characteristic length $L$ (such as the core cavity height which is about $2 \mathrm{~m}$ ), a fuel salt velocity $U$ (about $4 \mathrm{~m} / \mathrm{s}$ for the axial flow velocity in the core cavity) and a temperature variation $\delta T$ (core inlet and outlet temperatures are approximately $650^{\circ} \mathrm{C}$ and $750^{\circ} \mathrm{C}$ respectively resulting in a temperature rise of $100^{\circ} \mathrm{C}$ ), then the error of this approximation can be estimated from:

$$
1=\frac{\left|\frac{D \rho}{D t}\right|}{|\rho \nabla \cdot \vec{U}|} \sim \frac{\left|u_{z} \frac{\partial \rho}{\partial z}\right|}{\left|\rho \frac{\partial u_{z}}{\partial z}\right|} \sim \frac{U \frac{\delta \rho}{L}}{\rho\left(\frac{\delta U}{L}\right)} \sim \frac{\frac{\delta \rho}{\rho}}{\frac{\delta U}{U}}
$$

where $u_{z}$ is vertical component of the salt velocity in the core cavity. In the case of $\mathrm{LiF}_{-} \mathrm{ThF}$, the salt density variation between the core inlet and outlet can be estimated from the following experimental correlation [21] : $\rho(T)=A T+B$ where $A=4983,56 \mathrm{~kg} / \mathrm{m}^{3}$ and $B=-0,882 \mathrm{~kg} /{ }^{\circ} C \mathrm{~m}^{3}$. Therefore the relative density variation $\delta \rho / \rho$, and the induced velocity changes $\delta U / U$, between the core cavity inlet and outlet is approximately $2 \%$ which is small in comparison to numerical error arising from the turbulence modeling or from the uncertainties on the thermodynamics properties of the salt. This velocity change is also small in comparison to the velocity gradients that one can found in the core cavity near the walls [21].

Nevertheless larger temperature gradients (and density variations) may exist in some specific accidental transients, for example those involving natural convection. In these cases, Boussinesq approximation could introduce a large error and thus a Low Mach flow model or a full compressible should be preferred. As mentioned in Section 3.2.3, an experiment involving the study of natural convection of a molten salt is planned in the framework of the SWATH experimental studies. Data obtained from this experiment will help to analyze the limits of the above approximations.

The Reynolds Averaged Navier Stokes (RANS) equations [14] are usually solved using a turbulence cloture model such as the RNG (ReNormalization Group) k-epsilon turbulence model. The equations of the turbulence cloture model allow calculating the turbulent kinetic energy and the turbulent kinematic viscosity terms of the above equations. The fuel salt heat source $S$ of Equation 5 includes the energy released by the fission reaction and the decay heat from the fission products and the actinides. Energy arising from the viscous forces dissipation is negligible with respect to the nuclear power. The fission energy source can be calculated using a MSFR neutronics model developed from a deterministic approach such as a multigroup diffusion code [1] or a quasi-static code [8], or from a stochastic approach [16] such as a Monte Carlo code (MCNP [4, 18] or SERPENT [17] codes) or from a hybrid approach such as the transient fission matrices method [15]. The geometry of the neutronics models ideally includes the full fuel circuit (core cavity, inlet and outlet channels and the HXs) and 
eventually the fertile blanket and the neutron reflectors. Details on the neutronics model are not given here but can be found elsewhere $[1,15]$.

As discussed in Section 1, delayed neutron precursors concentrations have to be calculated in order to determine the reactor response. If one assumes that some phenomena such as precipitation or segregation are not significant, then the concentration of delayed neutrons precursors can be calculated by a transport of a chemical specie equation with two additional terms to take into account the precursors production rate from nuclear fission and the precursors decay rate [7]:

$$
\frac{\partial C_{l}}{\partial t}+\frac{\partial C_{l} \overline{u_{j}}}{\partial x_{j}}=D_{\text {prec }} \frac{\partial}{\partial x_{k}} \frac{\partial C_{l}}{\partial x_{k}}-\lambda_{l} C_{l}+\beta_{l} \nu_{t} \Sigma_{f} \phi \quad \text { for } l=1,2, \ldots, 8
$$

where $C_{l}$ is the delayed neutron precursors family ${ }^{8}$ (or group) $l, \overline{u_{j}}$ the time-averaged j-component of the flow velocity, $D_{\text {prec }}$ is the precursors diffusion coefficient in the salt, $\lambda_{l}$ the decay constant of the family- $l$ of delayed neutrons, $\beta_{l}$ the delayed fraction of the family- $l$ of delayed neutrons, $\nu_{t}$ the total number of neutrons produced by fission, $\Sigma_{f}$ the total fission macroscopic cross-section and $\phi$ the total neutron flux.

The set of Equations 3, 4, 5 and 7 provides a good description of the fuel salt flow in many operating conditions of the molten salt reactor. Nevertheless the accuracy of these equations depends upon the following assumptions:

(1) Validity of the incompressible flow with Boussinesq approximation: salt density variations are neglected in all the equations except in the gravity force term of Equation 4 which is replaced by a buoyancy force depending on the salt expansion coefficient $\beta_{\text {salt }}$. As already discussed, the Boussinesq approximation is valid if pressure-compressibility effects are small and also the density changes caused by temperature variations alone remain small,

(2) No change of phase (no salt solidification or melting),

(3) Single phase flow (bubbles arising from fission products are not considered),

(4) Turbulence cloture models and wall functions ${ }^{9}$, developed in many cases from experimental data for water, are considered valid for molten salts ${ }^{10}$,

(5) Heat transfer in the salt: only conduction and convective heat transfer mechanisms are considered.

Unfortunately some of these hypotheses can be questionable and may introduce large errors when studying the reactor behavior outside the range of nominal conditions. In particular these approximations may fail during accidents where very high temperatures, large temperatures gradients and/or salt phase change (solidification and melting) exist. The thermal hydraulics MSFR model requires thus further improvements (and experimental data) to overcome these limitations as it is discussed in the next section.

\section{Current Challenges Related to Molten Salt thermal hydraulics}

Current challenges related to molten salt thermal hydraulics can be grouped in two areas: (a) Modeling of the molten salt phenomena, (b) Developing experimental techniques adapted to molten salts.

\subsection{Modeling of the molten salt phenomena}

Accurate modeling of the behavior of the Molten Salt Fast Reactor requires the use of a multiphysics approach which takes into account the coupling existing between the relevant neutronics, thermal-hydraulics and thermomechanics phenomena in the reactor components. Two main strategies for developing a multiphysics model are possible. The simplest one uses a multiple-domain approach (or black box coupling) which couples

\footnotetext{
${ }^{8}$ For simplicity delayed neutrons precursors are represented by six to eight delayed neutron families (or groups) $l$, whose yields $\left(\beta_{l}\right)$ and decay constants $\left(\lambda_{l}\right)$ are obtained from experimental measurements. Delayed neutron groups are defined based on their half-lives. Eight delayed neutron families provided a better description for a fast spectrum reactor such as the MSFR.

${ }^{9}$ In the RANS approach, wall-functions provide simplified laws for the flow dimensionless velocity and temperature close to a wall in function to the dimensionless wall distance.

${ }^{10}$ Molten salts have slightly different Prandtl number $\operatorname{Pr}$ than liquid water at room conditions.
} 
independent single-physics models (i.e. the neutronics, the thermal hydraulics and the thermomechanics models). In the multiple-domain approach, data from the solvers of the various single-physics models is exchanged during the numerical resolution in order to converge to the solution. A different strategy, potentially more efficient but less flexible, is the single domain approach which uses only one numerical solver to solve all together the differential equations of the different models. Unfortunately this approach is not well adapted to our case because of the complexity of the physics models required for nuclear reactor analyses (e.g. the neutron transport problem and the Reynolds Averaged Navier-Stokes' equations). The more flexible multiple-domain strategy has therefore been adopted: fission rates are predicted using a neutronics code (for example a Monte Carlo code such as SERPENT or MCNP) and the salt thermal hydraulics equations are separately resolved using the CFD code OpenFOAM. Due to the complex interactions between the phenomena described by each of the single physics models, especial attention has to be given to the numerical aspects of the coupling schema to ensure that adequate convergence and stability of the solution is obtained. Evaluation of the propagation of the input data and numerical model uncertainties in the results is also necessary.

Regarding the thermal hydraulics model, and as it has been discussed in Section 1, only a CFD model provides sufficient accuracy for the calculation of the fuel salt velocity and temperature fields necessary to determine the cavity wall temperatures, the reactivity feedback coefficients and the concentration of the delayed neutrons precursors. Although a significant experience has been gained in the use of CFD models for nuclear applications in the past twenty years, further progress on the use of CFD models for molten salt coolants is needed. The CFD aspects that require particular attention are the selection of RANS models and wall functions [12] which ideally should be done based on the experimental data obtained for similar configurations.

As discussed in the previous sections, the flow structure in the MSFR core cavity is rather complicated since its shape (schematized in Figure 1) and the typical Reynols numbers induce a strong 3-D turbulent flow with possible recirculation zones [21]. Nevertheless the cavity geometry is relatively simple in comparison to typical geometries found in the core of other reactor concepts. For example Pressurized Water Reactor (PWR) fuel assemblies have a rather complicate geometry due to the presence of fuel rods, grids, upper and bottom nozzles. In this case, adequate discretization of the coolant flow in the boundary layer requires the use of a very large mesh containing tens of millions of cells per fuel assembly. On the contrary the MSFR core cavity can be resolved using a mesh with a smaller number of cells as can be seen in the example illustrated in in Figure 5. This CFD mesh is used to resolve the RANS approach equations and contains about 300,000 cells. The relative low number of cells of the MSFR CFD mesh makes possible to perform reactor transient calculations coupled to a neutronics model with a reasonable computational cost. Nevertheless if one wants to extend the CFD model to the entire fuel circuit difficulties arise at the heat exchangers (HXs). This reactor component has a large contact surface with the fuel salt to maximize the heat exchange. The discretization of the boundary layer in the HXs requires the use of a mesh with a large number of cells and thus significantly penalizes the numerical simulation. If one is interested in studying the phenomena in the core and determining the overall reactor response then some precision can be resigned in the HXs. The use of a porous medium approach [13] to model the HXs appears in this case as a convenient strategy to reduce the mesh requirements in the HXs while keeping a reasonable accuracy. Figure 6 illustrates the overall porous medium approach in which a heterogeneous geometry (fuel rods or pellets, HXs tubes or plates, etc.) is replaced by a continuous homogenous medium having equivalent macroscopic properties such as the porosity, permeability and conductivity tensor, etc. The main advantage of the porous approach is the strong reduction of the numerical discretization requirements and thus the computational cost. One of the drawbacks is that the complexity of the problem is shifted to the determination of the porous medium properties. These properties (for example the permeability and conductivity tensors or the heat exchange coefficient between the solid and fluid phases) are necessary to solve the porous medium equations. In practice due to the complexity of the microscopic phenomena, these properties cannot be rigorously derived from the mathematical homogenization of the mass, momentum and energy conservation equations at the microscopic level. Instead the porous medium properties are often obtained after introducing empirical or semi-empirical cloture laws to simplify the homogenization. In addition, the porous medium model provides only volume averaged values for the velocity and temperatures. Averaged values are calculated in an elementary volume 


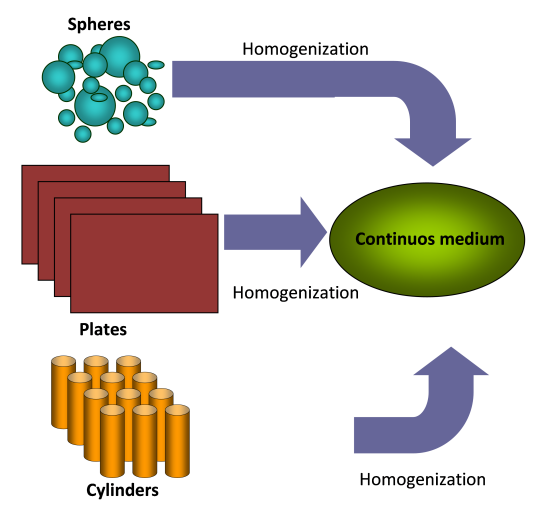

FIGURE 6. In a porous medium approach, the medium heterogeneities are homogenized and transformed in a continuous medium with averaged properties (porosity, permeability and conductivity tensors).

that has to be large enough to allow performing a meaningful averaging process (in a periodic system this means several unit cells). The microscopic (local) information could be in theory retrieved but in practice is difficult to obtain. One important hypothesis of a porous medium model is that the local (or microscopic) temperature variations in each phase of the porous medium (solid or liquid phases) have to be smaller that the macroscopic variations. Averaged parameters thus fail to provide accurate values close to the boundaries of the porous medium unless corrections for the discontinuity of the medium properties at the boundary are made. The porous medium properties have to be calculated prior to the numerical simulation based on experimental data or from detailed numerical models. In spite of these drawbacks, the use of a porous medium approach to model some of the reactor components such as the HXs provides, in general, a good compromise between precision and computational cost.

Another interesting challenge in modeling a molten salt reactor arises from the fact that working temperature of molten salts being considered for nuclear reactor applications are usually above several hundred of degrees Celsius. In particular in the current design of the MSFR the averaged temperature of the fuel salt is approximately $725^{\circ} \mathrm{C}$. Moreover during accidental conditions, the salt temperature could increase well beyond this value. At very high temperatures, the heat transfers mechanisms become quite complex and include heat conduction, convection and thermal radiation in the salt. Indeed the available experimental data [25] suggest that some molten salts should be considered as a semi-transparent medium [3] having a more or less complicate structure of optical bands. As discussed in section 1, radiative heat transfer in the fuel salt (liquid and solid phases) is currently not considered in Equation 5 but should be taken into account in the multiphysics model in particular for high temperature accidents of the MSFR. Unfortunately determination of the radiative heat transfer contribution of the fuel salt is quite challenging since the salt optical coefficients are not well known. In addition, the salt optical coefficients in the reactor may significantly change overtime as result of the salt composition shift due to irradiation effects and the density of particles in suspension. In despite of these challenges, various approaches to include radiative heat transfer in the energy conservation equation are being considered. The more adapted radiative heat transfer model[22] is schematized in Figure 7. This model approximates the fluid phase optical properties by two regions:

(a) Inside the band: the fluid phase is considered as an optically thick medium (i.e. a black band). Therefore radiative heat transfer inside the band can be considered as a diffusive process in which radiative heat flux is proportional to the fluid temperature gradient and thus it can be taken into account by a correction on the thermal conductivity of the salt.

(b) Outside the band: the fluid phase is considered as a transparent medium and thus radiative heat exchange takes place directly between the surfaces of the solid phase (which are assumed to be opaque). Modeling 


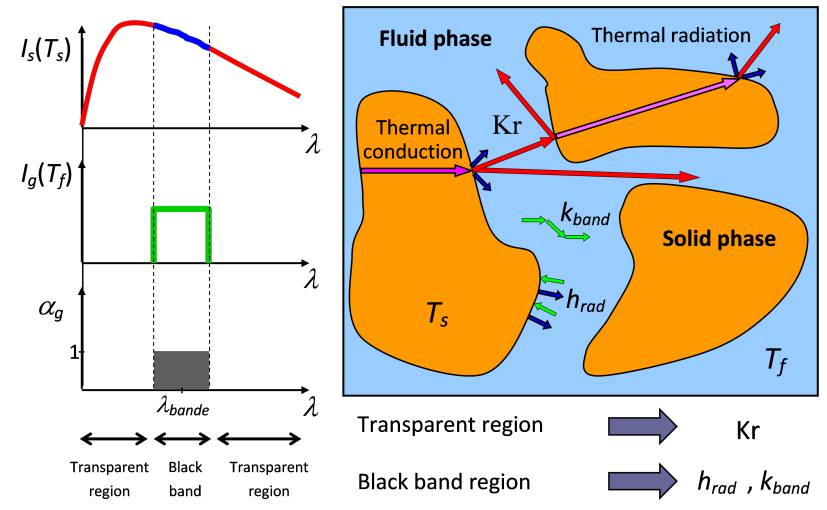

FiguRE 7. Example of radiative heat transfer modeling for a semitransparent fluid having one black band.

radiative heat transfer outside the band requires adding a new term in the energy conservation equation to account for the thermal radiative transport.

This approach can be adapted in function of the optical properties of the molten salt (e.g. number of bands) but due to the lack of accurate optical properties, more experimental data is necessary for a practical implementation. It is expected that some of the SWATH experiments could contribute to make further progress on the radiative heat transfer modeling.

Another important phenomenon that needs to be addressed is salt solidification/melting. Indeed unwanted fuel salt solidification in the MSFR components cannot be excluded during some normal and abnormal transients. For example during an hypothetical over-cooling accident the molten fuel salt flow may freeze inside the HXs as results of excessive heat removal in the intermediate circuit. The presence of partial or total blockages in the fuel circuit will affect the salt circulation and the heat transport. The inclusion of the salt phase change phenomena in the multiphysics model in therefore necessary. Moreover, an appropriate solidification model should fulfill the following requirements: (a) allow for the determination of the solidification (or melting) interface and (b) predict the solid phase micro-structure and properties. This later point is necessary since the behavior of a reactor components containing a fraction of solidified salt depends on the thermomechanical properties of the solid salt phase (for example the thermal conductivity, mechanical resistance, porosity and residual heat). As can be seen in Figure 8, the salt solid phase structure could be quite complex and depends on solidification process conditions (such as the velocity of the solidification interface, the undercooling temperature, the fraction of dissolved gases, salt composition, etc.). Accurate modeling of the salt solidification with reasonable computation cost is quite challenging but an approach combining microscopic [9, 24] and macroscopic [2, 5] modeling of the mushing zone could be used and it is currently being investigated. Alternatively, an Multi-scale solidification approach to this problem is also possible [20].

Finally it is worth to note that during normal conditions the molten salt flow can be considered as an incompressible (low Mach number) flow. However, during some hypothetical accidental transients involving a sudden large reactivity insertion, pressure-compressibility effects may no longer be negligible and would require an evaluation using a compressible flow model.

\subsection{Developing experimental techniques for molten salts}

Developing experimental facilities to investigate molten salt flows is quite challenging because of the high working temperatures, the chemical reactivity of the salts and the lack of adapted instrumentation. In the particular case of fluorides salts, for example a LiF-NaF-KF eutectic (called here FLiNaK for simplicity), to avoid unwanted salt solidification as can be seen in Figure 9, active electric heaters and adapted thermal insulation have to be added on the exterior walls of all the components of the experimental facility. This imposes 


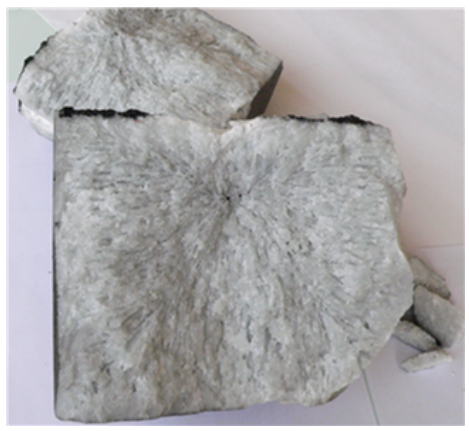

Figure 8. Complex structure of a solidified LiF-NaF-KF eutectic (FLiNaK).

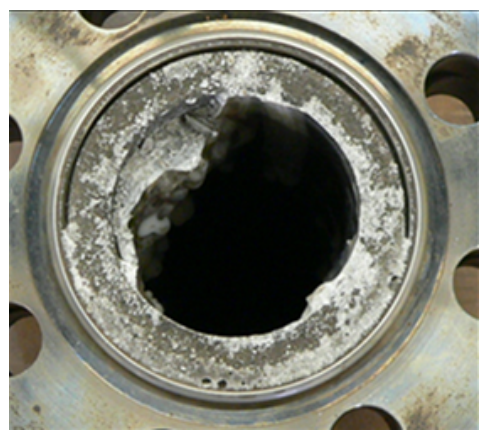

Figure 9. Salt solidification in the inside a pipe of a FLiNaK experiment.

significant burden on the mechanical design of the pipes, tanks, vanes, etc. and also if repairs or inspection of a components is needed. Another drawback of fluorides molten salts is their chemical reactivity to water vapor which requires the use of inert atmosphere. In case of an accidental salt leak and subsequent exposure to air, salt corrosion on stainless steel mass will progress very fast leading to a catastrophic failure of the component. As an example Figure 10 illustrates a case of corrosion damage due to a small leak of salt in a welding between a salt pipe and a gas injector of the FFFER loop.

Thermohydraulics measurements on a molten salt requires the use of adapted instrumentation. Flow and level measurements of a high temperature fluid are difficult and very often demand to develop new instrumentation. Implementation of classical flow visualization techniques using lasers is not practical for high temperatures experiments in close channels since a transparent windows induces a thermal perturbation in the flow. Section 3.2 discusses on a solution to partially overcome this problem.

\section{Ongoing Experimental and Modeling Developments}

Significant experience has been gained at CNRS on molten salt experiments design and operation. This section presents two key experiments designed to study specific molten salt phenomena relevant to the Molten salt fast reactor (MSFR): (a) FFFER and (b) SWATH facilities.

\subsection{Forced Fluoride Flow for Experimental Research (FFFER)}

The FFFER facility is a LiF-NaF-KF eutectic (FLiNaK) salt force convection loop whose main objectives are: (a) Study the bubble separation process in the salt and (b) Acquire technical experience in designing and operating high temperature salt experiments. As can be seen in Figure 11 the main components of the loop are the molten salt circulator, the bubble separator device, the salt tank, the gas injectors (argon), the loop pipes 


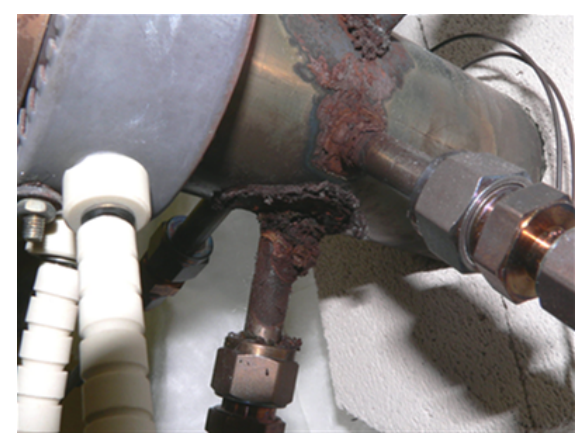

Figure 10. Example of corrosion damage in a LiF-NaF-KF eutectic (FLiNaK) loop component.

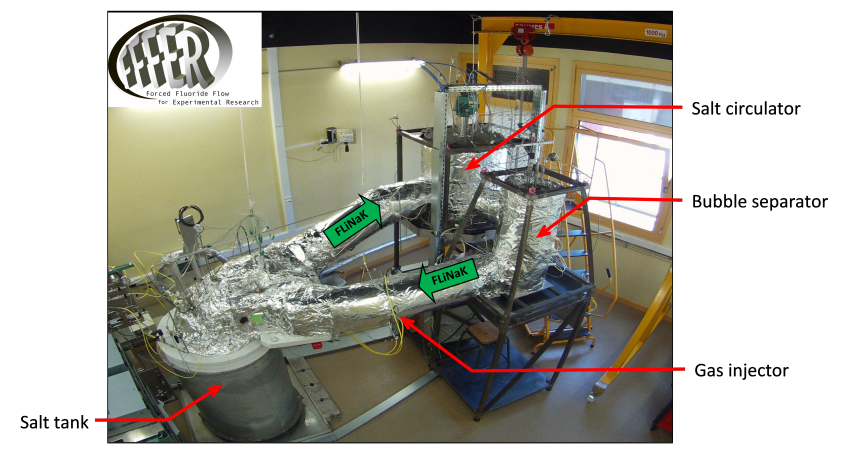

FiguRE 11. FFFER - Force Fluoride Flow for Experimental Research (FLiNaK force convection loop at LPSC Grenoble).

and vanes (including a cold plug device). All the loop components have to be insulated to reduce the heat losses and contain electrical heaters to maintain the salt above the solidification point. FFFER loop components are made of SS 304L.

The loop contains gas (argon) injectors and a bubble separator to study the performance of the bubbling process. This device is intended as an online MSFR reprocessing system that would allow for partial extraction of the fission products in the MSFR, in particular for the gaseous fission products. Extraction of part of the fission products from the MSFR fuel circuit allows decreasing the parasitic neutron absorptions and improving the corrosion control. In FFFER, the bubble separator has been designed to induce a strong rotation of the molten salt flow inside this component which allows for separation between gas bubbles and the molten salt by the effect of the centripetal force and the density differences (argon is extracted from the center of the separator). By comparing the gas flow (argon) between the gas injector and the bubbles separator, the overall efficiency of the bubbles separator can be determined.

Another novel component of FFFER is a cold plug device (not visible in Figure 11) which is used as safety device in FFFER in a similar manner as in the reactor: the cold plug is designed to passively melt in case of a loss of electric power. When the cold plug melts the molten salt in the loop flows by gravity forces to the salt tank where salt is usually stored. The working principle of the cold plug relies on the passive control of the heat transfer balance inside the device which determines whether the salt inside the cold plug solidifies or melts. In the MSFR, the cold plug device fulfills a similar function. During nominal operation of the MSFR the salt inside the cold plug should remain in solid phase (the cold plug vane is closed). In the case of an accident (for example a loss of electrical power) the resulting local heat unbalance should lead to a rapidly salt 
melting which would allow for passive fuel salt draining into the salt draining tanks (the cold plug vane is then open). The fuel salt draining tanks should be designed to ensure that adequate margins are obtained regarding the fuel cooling capabilities and the reactivity control (these are two key safety functions in a nuclear reactor). A careful evaluation of the performance of the cold plug is therefore required for the reactor safety analyses. In particular, the cold plug characteristic time for total melting is a key parameter since the fuel salt should be drain out from core cavity in a few minutes to avoid excessive temperature rise due to nuclear decay heat. FFFER facility has been used to test a preliminary design of a cold plug device. These preliminary tests suggest that it is possible to design this component in order to meet the time limit for the core draining. More work is however required to show that the melting will quickly start in all possible scenarios.

While the experimental measurements in FFFER facility are not finished some key results can already be highlighted. In particular, the preliminary results shown a very good performance of both the bubble separator and the cold plug devices. In addition to this valuable experimental data, FFFER facility has fulfilled the objective of providing valuable design and operation experience in areas such as molten salt instrumentation, flow control system, heating and thermal insulation, molten salt vanes and salt circulator. This technical experience is keystone for the success of future experiments with molten salts in particular for the design and construction phase of the SWATH facility that is discussed in the next section.

\subsection{Salt at WAll: Thermal ExcHanges (SWATH)}

The Salt at WAll: Thermal ExcHanges (SWATH) experiment is one of the research activities of the European H2020 SAMOFAR project. The main objectives of SWATH are to: (i) Improve molten salt numerical models used for design and safety studies and (ii) Demonstrate the working principles of the cold plug device and study various design options. A critical point of SWATH is to ensure that the experimental data uncertainties are significantly lower than the effects of the thermal hydraulic phenomena that are being studied. Since the possible accidental configurations of the MSFR reactor (geometries and conditions) are not completely known, SWATH is focused on understanding the underlying physical principles rather than developing experimental correlations. To begin with the experiment design, a Phenomena Identification Ranking Table (PIRT) analysis was used to sort out the thermal hydraulics fuel salt phenomena according to their importance with respect to the reactor studies. This analysis led to the following ranking list:

(1) Heat transfer in very simple geometries,

(2) Evolution of the salt solidification interface with and without forced convection,

(3) Solidification along a cold wall after successive molten salt flows (lava flow like),

(4) Flow structure characteristics in an open channel,

(5) Turbulence effects on the flow velocity and temperature profiles near a wall and

(6) Radiative heat transfer in the salt.

The SWATH experimental setup will have two main elements: (a) the SWATH facility which allows establishing a controlled flow and (b) the test sections ${ }^{11}$ where the measurements are made. The design of both elements (the facility and the test sections) poses several challenges due to the high working temperatures, the salts chemical reactivity with water vapor and the lack of adapted instrumentation. As it is discussed in this section the same fluoride salt (a LiF-NaF-KF eutectic) used in the FFFER experiment has been selected as working fluid for SWATH. Due to the high melting point of fluoride salts, the experiment setup needs the use of active electric heaters and adapted thermal insulation to avoid salt solidification on the inner wall of the components. Since fluorides salts reacts with water, a glovebox filled with an argon gas is also required to host the test sections including the instrumentation and the handling devices. The glovebox will contain the test sections during the experiment and will allow for performing post-test measurements without putting them back into air.

High temperatures and risk of chemical reactions make hydraulics measurements quite challenging, in particular performing flow rate and liquid level measurements. Under these conditions implementation of classical

${ }^{11}$ The test sections are very simple geometries that will be used to investigate the salt phenomena. 


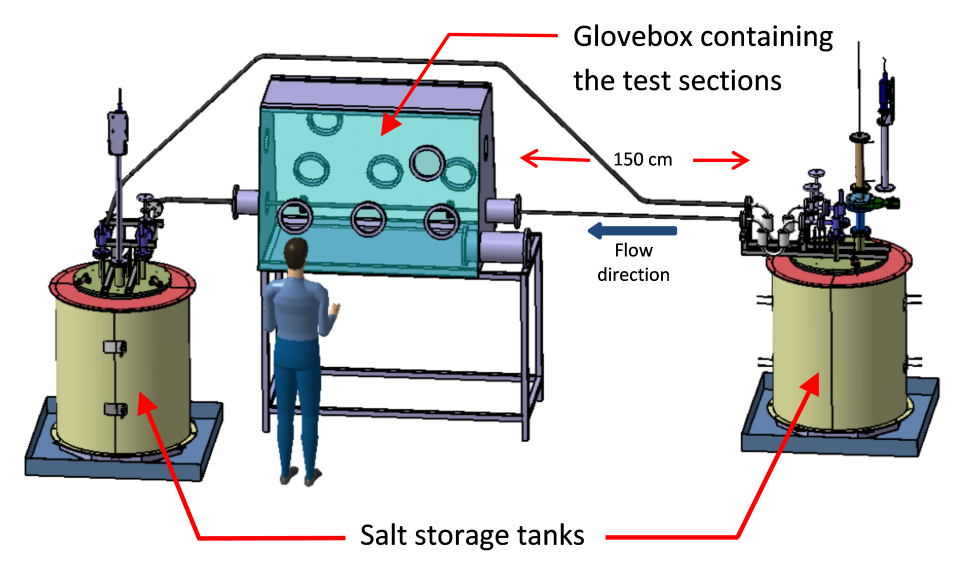

Figure 12. Approximate layout of the future Salt at WAll: Thermal ExcHanges (SWATH) facility.

flow visualization techniques such as PIV (Particle Image Visualization) is not practical. To overcome some of these difficulties two separate facilities will be built: SWATH-W using water as working fluid and SWATH-S using a fluoride salt. Since PIV can be implemented in SWATH-W, this facility will be used to study the accuracy of the CFD models predictions regarding the flow velocity field. Indeed kinematic viscosity is very similar between LiF-ThF4 and water as can be seen in the Table 1. The SWATH-S facility will be focused instead on the investigation of the heat transfers aspects of the models.

The operation of both SWATH facilities is based on a discontinuous working principle in which the flow is established by regulating the pressure difference between two tanks, rather than using a pump. Figure 12 presents an sketch of the SWATH-S facility which is composed by the two salt storage tanks, the pipes, with the heating system and the thermal insulation (not shown in the figure), the glovebox where the test section is placed under an argon atmosphere and the control system and the experiment instrumentation. The pressure control system is designed to maintain a stable flow during the operation of the loop by regulating opening and closing of tank vanes connected to a pressurized argon tank and the atmosphere. This control system uses information related to the flow rate such as the pressure drop measured at specific loop components and also the salt level in the tanks. Salt mass flow rate is calculated from the variation of the tank levels measured by two independent methods: a laser beam system and electrical contactors system.

\subsubsection{SWATH-W (water facility)}

The SWATH-W facility uses water at nearly room conditions (pressure and temperature) and has various purposes:

- Performing purely hydraulic measurements: the low temperature and pressure working conditions of SWATH-W make possible to build the test sections on Plexiglas. This allows performing PIV (Particle Image Visualization) measurements to precisely determine the flow conditions inside the test section. Some of the test section geometries will be studied both in SWATH-W and SWATH-S. For these particular geometries the PIV data from SWATH-W will be used to verify the accuracy of the CFD models at isothermal conditions while the thermal effects will be investigated in SWATH-S. The flow field measurements in SWATH-W will also serve to confirm that true fully developed flow conditions are obtained at the entrance of the test sections.

- Experiment design support: SWATH-W will be used to evaluate experiment design options before building or modifying the SWATH-S facility or the test sections. Furthermore SWATH-W will serve to confirm that the pressure control system allows obtaining adequate steady conditions in the test 


\begin{tabular}{|c|c|c|c|c|c|}
\hline Fluid & $\begin{array}{c}\text { Temperature } \\
{\left[{ }^{\circ} \mathrm{C}\right]}\end{array}$ & $\begin{array}{c}\text { Density } \\
{\left[\mathrm{kg} / \mathrm{m}^{3}\right]}\end{array}$ & $\begin{array}{c}\text { Heat Capacity } \\
{\left[\mathrm{kJ} / \mathrm{m}^{3} \mathrm{~K}\right]}\end{array}$ & $\begin{array}{c}\text { Thermal Conductivity } \\
{[\mathrm{W} / \mathrm{mK}]}\end{array}$ & $\begin{array}{c}\text { Kinematic Viscosity } \\
{\left[\mathrm{m}^{2} . \mathrm{s}\right]}\end{array}$ \\
\hline $\mathrm{LiF}_{\mathrm{ThF}}$ & 900 & 3948 & 8489 & 1,03 & $1,2910^{6}$ \\
\hline $\mathrm{H}_{2} \mathrm{O}$ & 20 & 998 & 4177 & 0,60 & $1,00.10^{6}$ \\
\hline
\end{tabular}

TABLE 1. Thermodynamics properties of $\mathrm{LiF}_{-} \mathrm{ThF}_{4}$ molten fuel salt at $900^{\circ} \mathrm{C}$ and water at room conditions $\left(20^{\circ} \mathrm{C}\right.$ and 1,01325 bar $)$.

section. The proper functioning of some other facility components will also be checked in SWATH-W (for example the siphon pipes and level measurement system).

- Aide to the definition of the experiment procedures: when it is possible, the experimental test procedures will be checked first in SWATH-W and then implemented in SWATH-S (for example determination of the time delay required after SWATH start-up to obtain steady flow in the test section).

\subsubsection{SWATH-S (molten salt facility and salt choice)}

The second facility, SWATH-S, is being designed to perform the high temperature thermal hydraulics experiments and investigate the accuracy of the salt models regarding heat transfers and phase change. Molten salts such as $\mathrm{LiF}_{-} \mathrm{ThF}_{4}$ (reference salt for the MSFR) have excellent heat storage capacities but less good thermal conductivity as can be seen in the Table 1. It follows that in molten salts convective heat transfer mechanism is more efficient than conduction in comparison to other coolants. The selection of the working fluid for SWATH-S requires making a compromise between practical experimental considerations and obtaining a similar thermal hydraulic behavior to the molten salt in the reactor.

The use of a molten salt with a relative low temperature melting point in SWATH-S was initially considered but finally abandoned because it would precluded the possibility of studying the effect of radiative heat transfer which is expected to play a significant role in some accidental conditions. The use of a lithium fluoride salt ( $\mathrm{LiF})$ was neither retained since it imposes a too high working temperature (melting point is at about $850^{\circ} \mathrm{C}$ ). Practical considerations (facility licensing for example) excluded also the possibility of employing a non-irradiated fuel salt (i.e. $\mathrm{LiF}_{-} \mathrm{ThF}_{4}$ ) which has a lower melting point (about $585^{\circ} \mathrm{C}$ ). A FLiNaK salt was finally found to be a good compromise since it allows achieving in SWATH-S a very good similitude with respect to the phenomena encountered in a Molten Salt Reactor. In addition FLiNaK has a high enough melting point (at about $450^{\circ}$ ) to allow for investigation of the effect of radiative heat transfer. On the other hand FLiNaK melting point is sufficiently low to avoid excessive complication on the facility design, construction and operation (for example SWATH-S components can be made on SS 304L).

Design of the experiment in order to obtain a reasonable similitude with the lithium fluoride fuel salt of the MSFR requires consideration of at least the three following dimensionless numbers:

- Reynolds Number: $R e=(\rho L U) / \mu$, to obtain similar flow regime (laminar or turbulent),

- Prandtl Number: $\operatorname{Pr}=\left(\mu C_{p}\right) / \lambda$, to maintain the relative importance between heat transfer mechanisms (convection versus conduction),

- Grashof Number: $G r=\beta \Delta T g L^{3}(\rho / \mu)^{2}$, when the effects of natural convection have to be considered.

Reynolds and Grashof numbers can be adjusted in SWATH-S to obtain reasonable similitude by changing the experimental setup characteristic length $L$ and the flow velocity $U$ (for the Reynolds number) and the $\Delta T$ (for the Grashof) in the test section. As can be seen in the example of Table 2, Reynolds numbers up to 20,000 can be obtained in the experiment assuming a characteristic length $L$ (hydraulic diameter) equal to $25 \mathrm{~mm}$ and a maximal flow velocity $U$ equal to $1 \mathrm{~m} / \mathrm{s}$. Higher Reynolds numbers could be obtained by further reducing the flow cross section (with same volumetric flow). The Reynolds number range of SWATH-S is not intended to cover all possible values existing in the reactor ${ }^{12}$. Nevertheless it will allow studying selected laminar and turbulent molten salt flow configurations that will help to improve the numerical modeling of the salt phenomena

\footnotetext{
${ }^{12}$ Maximum Reynolds number in the MSFR is about 500,000 inside the core cavity at nominal conditions.
} 


\begin{tabular}{|c|c|}
\hline \multicolumn{2}{|c|}{ FLiNaK } \\
\hline $\mathrm{T}\left[{ }^{\circ} \mathrm{C}\right]$ & Re \\
\hline 475 & 5200 \\
\hline 500 & 6100 \\
\hline 525 & 7200 \\
\hline 550 & 8400 \\
\hline 575 & 9700 \\
\hline 600 & 11000 \\
\hline 625 & 12500 \\
\hline 650 & 14000 \\
\hline 675 & 15700 \\
\hline 700 & 17400 \\
\hline
\end{tabular}

TABLE 2. FliNaK Reynolds numbers assuming a characteristic length $L$ equals to $25 \mathrm{~mm}$ and a flow velocity $U$ equals to $1 \mathrm{~m} / \mathrm{s}$.

\begin{tabular}{|c|c|c|c|c|c|}
\hline \multicolumn{2}{|c|}{$\mathrm{LiF}_{-} \mathrm{ThF}_{4}$} & \multicolumn{2}{|c|}{ FLiNaK } & \multicolumn{2}{|c|}{ Hitec } \\
\hline $\mathrm{T}\left[{ }^{\circ} \mathrm{C}\right]$ & Prandtl & $\left.\mathrm{T}\left[{ }^{\circ} \mathrm{C}\right]\right]$ & Prandtl & $\mathrm{T}\left[{ }^{\circ} \mathrm{C}\right]$ & Prandtl \\
\hline 600 & 21,0 & 475 & 24,0 & 175 & 27,6 \\
\hline 700 & 16,0 & 500 & 20,0 & 200 & 22,8 \\
\hline 800 & 12,8 & 525 & 16,8 & 225 & 18,8 \\
\hline 900 & 10,6 & 550 & 14,3 & 250 & 15,4 \\
\hline 1000 & 9,1 & 575 & 12,3 & 275 & 12,6 \\
\hline 1100 & 7,9 & 600 & 10,7 & 300 & 10,4 \\
\hline 1200 & 7,1 & 625 & 9,3 & 325 & 8,6 \\
\hline 1300 & 6,4 & 650 & 8,2 & 350 & 7,1 \\
\hline & & 675 & 7,2 & 375 & 6,1 \\
\hline & & 700 & 6,5 & & \\
\hline
\end{tabular}

TABLE 3. LiF-ThF4, FliNaK and Hitec molten salts Prandtl numbers at various temperatures. As an example, temperature range of the MSFR fuel salt is expected to be comprised between $600^{\circ} \mathrm{C}$ and $1300^{\circ} \mathrm{C}$ during most normal and accidental situations.

described in previous sections. Moreover, some of the low Reynolds configurations studied in SWATH-S are of interest during accidental conditions (for example during a Loss Of Flow accident) inside specific reactor components (such as HXs or the draining system). Studying the molten salt flow in a experimental model having both geometric and dynamic (i.e. at very high Reynolds) similarities to those of the reactor cavity will be necessary at a later stage of the reactor design. This is not needed for the actual purpose of this work which is focused on the thermal hydraulics phenomena modeling. The Prandtl Number $(P r)$ depends only on the fluid intrinsic characteristics which in our applications are function of the temperature. As can be seen in the example of Table 3, by changing the FLiNaK temperature between $500^{\circ}$ and $700^{\circ}$ the likely normal and accidental fuel salt flow Prandtl number can be adequately covered.

The salt tanks volume in SWATH-S has been sized to store about 60 liters of molten salt which allows establishing a steady flow for several minutes (between 10 to 30 minutes) depending on the volumetric flow rate. Salt mass flow rate is calculated in SWATH-S from the variation of the tank levels measured by two independent methods: a laser beam system and electrical contactors system. As can be seen in Figure 12, the pipe connecting the salt tank and the experimental section has been designed to form a straight channel of more than $150 \mathrm{~cm}$ to obtain fully developed flow conditions before the entrance to the test section. To reduce the 
mechanical stresses due to thermal expansion during the experiments the facility use deformable connections, pipe bends and free moving components. All components are made on stainless-steel (SS 304L).

\subsubsection{SWATH test sections}

SWATH test sections geometries are designed to study different salt phenomena. Only simple geometries will be used to minimize possible sources of errors and uncertainties in the experiments and also in the numerical models employed to model the experiments. The test sections can be divided into two groups:

- Test sections used to perform molten salt phase change experiments

- Test sections used to perform heat exchange experiments in closed or open channels.

The goal of the phase change experiments is the evaluation of the accuracy of the microscopic and macroscopic salt solidification models. Some of these experiments include solidification of a molten salt pool with natural or forced convection and solidification of a molten salt over a "cold" surface with force convection. The properly functioning of the cold plug device will also be investigated in this first group of experiments. The second group of experiments will be used to investigate the heat transfer between the salt and the walls. These experiments will be performed using open and close channels with circular or rectangular section. Some of the tested sections will be built on Plexiglas and studied in SWATH-W to obtain a detail flow field data and to check the precision of the CFD models predictions. Data obtained from the test sections will be used to investigate the accuracy of the solidification, turbulence and radiative heat transfer models for the fuel salt and also to provide general recommendations on the salt thermal hydraulic modeling.

\section{Perspectives}

An overview of the ongoing efforts to improve the understanding of molten salt thermal hydraulics phenomena has been presented. The current status of the design of the experimental facility SWATH (Salt at WAll: Thermal ExcHanges) was also discussed. This facility is being built as part of the European project SAMOFAR (20152019). In parallel to the experimental work, part of the effort is focused in the development of more accurate solidification, turbulence and radiative heat transfer models for the fuel salt. Experimental data measured from the SWATH experiment will be used to investigate the accuracy of these advanced numerical salt models. Comparison between the experiment results and model predictions is expected to start in 2017. Integration of the new salt models in the MSFR multiphysics code will follow and the updated reactor model will be used for the evaluation of the MSFR reactor behavior under a larger range of normal and accidental conditions than in the existing studies.

\section{REFERENCES}

[1] M. Aufiero. Development of advanced simulation tools for circulating fuel nuclear reactors. PhD thesis, Politecnico di Milano, 2014.

[2] A. Bhattacharya, A. Kiran, S. Karagadde, and P. Dutta. An enthalpy method for modeling eutectic solidification. Journal of Computational Physics, 262(1):217-230, april 2014.

[3] M. Q. Brewster. Thermal radiative transfer and properties. John Wiley \& Sons Inc., 1992.

[4] J. F. Briesmeister ed. Mcnp-a general monte carlo code for neutron and photon transport, version 3a. Technical Report LA-7396-M, Los Alamos National Laboratory, september 1986.

[5] J. Dantzig and M. Rappaz. Solidification. EPFL Press, 1 edition, april 2009.

[6] S. Delpech, E. Merle-Lucotte, D. Heuer, M. Allibert, V. Ghétta, C. Le-Brun, X. Doligez, and G. Picard. Reactor physic and reprocessing scheme for innovative molten salt reactor system. Journal of fluorine chemistry, 130(1):11-17, january 2009.

[7] J. J. Duderstadt and L. J. Hamilton. Nuclear Reactor Analysis. John Wiley \& Sons Inc., New York, 1976.

[8] S. Dulla, E. H. Mund, and P. Ravetto. The quasi-static method revisited. Progress in Nuclear Energy, 50(8):908-920, november 2008. 
[9] B. Gonzalez-Ferreiro, H. Gomez, and I. Romero. A thermodynamically consistent numerical method for a phase field model of solidification. Communications in Nonlinear Science and Numerical Simulation, 19(7):2309-2323, july 2014.

[10] H. Jasak, A. Jemcov, and Z. Tukovic. Openfoam: A c++ library for complex physics simulations. In International workshop on coupled methods in numerical dynamics, Dubrovnik, Croatia. IUC, september 2007.

[11] I. Jasak. Openfoam reference guide. http://www.openfoam.org/docs/.

[12] G. Kalitzin, G. Medic, G. Iaccarino, and P. Durbin. Near-wall behavior of rans turbulence models and implications for wall functions. Journal of Computational Physics, 204(1):265-291, march 2005.

[13] M. Kaviany. Principles of heat transfer in porous media. Springer Science \& Business Media, 1999.

[14] P. K. Kundu, I. M. Cohen, and D. R. Dowling. Fluid mechanics. Academic Press, 2011.

[15] A. Laureau, M. Aufiero, P. R. Rubiolo, E. Merle-Lucotte, and D. Heuer. Coupled neutronics and thermalhydraulics transient calculations based on a fission matrix approach: application to the molten salt fast reactor. In Joint International Conference on Mathematics and Computation, Supercomputing in Nuclear Applications and the Monte Carlo Method (MEC + SNA + MC 2015), Nashville, USA. American Nuclear Society, march 2015.

[16] A. Laureau, P. R. Rubiolo, D. Heuer, E. Merle-Lucotte, and M. Brovchenko. Coupled neutronics and thermal-hydraulics numerical simulations of a molten fast salt reactor (mfsr). In $S N A+M C 2013$ - Joint International Conference on Supercomputing in Nuclear Applications + Monte Carlo. EDP Sciences, 2014.

[17] J. Leppänen. Serpent - a Continuous-energy Monte Carlo Reactor Physics Burnup Calculation Code. VTT Technical Research Centre of Finland, june 2015. An optional note.

[18] Los Alamos National Laboratory. MCNP website. http://mcnp.lanl.gov.

[19] L. Mathieu, D. Heuer, E. Merle-Lucotte, R. Brissot, C. Le-Brun, E. Liatard, J.-M. Loiseaux, O. Méplan, A. Nuttin, and D. Lecarpentier. Possible configurations for the thorium molten salt reactor and advantages of the fast nonmoderated version. Nuclear Science and Engineering, 161(1):78-89, january 2009.

[20] T. Pitkänen, S. Majaniemi, and T. Ala-Nissila. Multiscale modelling of microstructure formation in polymer casting. Technische Mechanik, 30(1-3):259-268, 2010.

[21] H. Rouch, O. Geoffroy, P. R. Rubiolo, A. Laureau, M. Brovchenko, D. Heuer, and E. Merle-Lucotte. Preliminary thermal-hydraulic core design of a molten salt fast reactor (MSFR). Annals of Nuclear Energy, 64:449-456, february 2014.

[22] P. R. Rubiolo and J.-M. Gatt. Modeling of the radiative contribution to heat transfer in porous media composed of spheres or cylinders. International Journal of Thermal Sciences, 41(5):401-411, april 2002.

[23] P. R. Rubiolo, D. Heuer, E. Merle-Lucotte, M. Brovchenko, V. Ghetta, M. Allibert, and A. Laureau. Overview and perspectives of the molten salt fast reactor (MSFR) concept. In Proceedings of the International Conference on Molten Salts in Nuclear Technology (CMSNT), Mumbai, India, january 2013.

[24] I. Steinbach. Phase-field models in materials science. Modelling and Simulation in Materials Science and Engineering, 17(7):073001, july 2009.

[25] P. T. Williams. Thermal radiative transport through lif for temperatures near the melting point. Technical Report DE88011586, Oak Ridge National Laboratory, TN (USA), june 1988. 